PROCEEDINGS OF THE

AMERICAN MATHEMATICAL SOCIETY

Volume 126, Number 11, November 1998, Pages 3413-3415

S $0002-9939(98) 04412-8$

\title{
ON THE CONTINUITY OF THE EVALUATION MAPPING ASSOCIATED WITH A GROUP AND ITS CHARACTER GROUP
}

\author{
GERHARD TURNWALD
}

(Communicated by Roe Goodman)

\begin{abstract}
For an abelian Hausdorff group $G$, let $G^{*}$ denote the character group endowed with the compact-open topology and let $\alpha_{G}: G \rightarrow G^{* *}$ denote the canonical homomorphism. We show that the evaluation mapping from $G^{*} \times G$ into the torus is continuous if and only if $G^{*}$ is locally compact and $\alpha_{G}$ is continuous. If $\alpha_{G}$ is injective and open, then the evaluation mapping is continuous if and only if $G$ is locally compact. Several examples and counterexamples are given.
\end{abstract}

Let $G$ be an abelian Hausdorff group. The characters of $G$ (i.e., the continuous homomorphisms from $G$ into $\mathbf{T}=\{z \in \mathbf{C}:|z|=1\}$ ) form a group $G^{*}$ under pointwise operations. If $G^{*}$ is endowed with the compact-open topology then it is an abelian Hausdorff group, called dual group (or character group) of G. (Cf. [8], [5], [1].) Evaluating characters at points of $G$ gives rise to a canonical homomorphism $\alpha_{G}: G \rightarrow G^{* *}$. The group $G$ is called reflexive if $\alpha_{G}$ is a topological isomorphism. The classical Pontryagin - van Kampen duality theorem states that every locally compact abelian group is reflexive. It was proved by M.F. Smith ([12]; cf. [1], $15.2)$ that the additive group of every Banach space is reflexive. Kaplan proved ([6]; [1],14.11) that every product of reflexive groups is reflexive. See [1] (and [10]) for further examples and background.

We use multiplicative notation and denote the unit element of $G$ by $e$. The group $G^{*}$ is an abelian Hausdorff group. The family of sets $P(K, V)=\left\{\chi \in G^{*}: \chi(K) \subseteq\right.$ $V$ \}, where $K$ is a compact subset of $G$ and $V \ni 1$ is an open subset of $\mathbf{T}$, forms an open neighbourhood base at the unit element $\chi_{0}$ of $G^{*}$. Taking into consideration that the mappings $x \mapsto \chi(x)$ and $\chi \mapsto \chi(x)$ are continuous homomorphisms, it is easily seen that the evaluation mapping $(\chi, x) \mapsto \chi(x)$ is continuous if it is continuous at $\left(\chi_{0}, e\right)$. Noting that $\chi(x) \in V$ for all $(\chi, x) \in P(K, V) \times K$, it follows that the evaluation mapping is continuous if $G$ is locally compact. If $G$ is locally compact, then $G^{*}$ is locally compact (cf. the Lemma stated below).

We employ the following well-known version of the Arzela - Ascoli theorem. It is an obvious consequence of [9],2.2 or [1],1.5; cf. also [8],Thm.9.

Lemma. Let $V=\{\exp (i t):|t|<\pi / 2\}$. If $U$ is a neighbourhood of e, then $P(U, V)$ has compact closure in $G^{*}$.

Received by the editors October 30, 1996 and, in revised form, March 21, 1997.

1991 Mathematics Subject Classification. Primary 22A05; Secondary 22D35.

Key words and phrases. Character group, reflexive group, evaluation mapping. 
Remark. Let $V_{1}=\{\exp (i t):|t|<2 \pi / 3\}$. Clearly, $\left\{z \in V_{1}: z^{2} \in V_{1}\right\} \subseteq V$. Hence $P\left(U^{2}, V_{1}\right) \subseteq P(U, V)$. Thus the Lemma implies that, for every neighbourhood $U$ of $e, P\left(U, V_{1}\right)$ has compact closure in $G^{*}$. The following example shows that this improvement of the Lemma is optimal.

If $G$ is an infinite compact group of exponent 3 (e.g., an infinite product of cyclic groups of order 3), then $\chi(x)^{3}=1$ for every $\chi \in G^{*}$ and $x \in G$. Thus $P\left(U, \overline{V_{1}}\right)=G^{*}$ is not compact (since $G^{*}$ is an infinite discrete group).

Theorem. The following properties are equivalent for every $G$ :

(i) The evaluation mapping $G^{*} \times G \rightarrow \mathbf{T},(\chi, x) \mapsto \chi(x)$, is continuous at $\left(\chi_{0}, e\right)$.

(ii) The evaluation mapping $G^{*} \times G \rightarrow \mathbf{T},(\chi, x) \mapsto \chi(x)$, is continuous.

(iii) $\alpha_{G}$ is continuous and $G^{*}$ is locally compact.

Proof. We have already noted above that (i) implies (ii). Let $V$ be a neighbourhood of 1 in T. If (ii) holds, then there exist open sets $U \ni e$ and $W \ni \chi_{0}$ such that $\chi(x) \in V$ for all $\chi \in W$ and all $x \in U$, hence $W \subseteq P(U, V)$. The Lemma implies that $W$ has compact closure if $V$ is sufficiently small. Thus $G^{*}$ is locally compact. If $B \subseteq G^{*}$ is compact, then (ii) implies that there is a neighbourhood $U$ of $e$ such that $\chi(x) \in V$ for all $(\chi, x) \in B \times U$. It follows that $\alpha_{G}(U) \subseteq P(B, V)$, and hence $\alpha_{G}$ is continuous. Thus (ii) implies (iii).

Suppose that (iii) holds. The evaluation mapping $G^{* *} \times G^{*} \rightarrow \mathbf{T}$ is continuous because $G^{*}$ is locally compact. Since $\alpha_{G}(x)(\chi)=\chi(x)$, the continuity of $\alpha_{G}$ implies (i).

Corollary. Let $G$ be an abelian Hausdorff group such that $\alpha_{G}$ is injective and open. Then the evaluation mapping $G^{*} \times G \rightarrow \mathbf{T}$ is continuous if and only if $G$ is locally compact.

Proof. Suppose that the evaluation mapping is continuous. The Theorem yields that $G^{*}$ is locally compact and $\alpha_{G}$ is an embedding. It follows that $G^{* *}$ is locally compact. Hence $\alpha_{G}(G)$, which is an open subset of $G^{* *}$, is locally compact. Thus $G$ is locally compact. The converse has already been noted above.

Remarks. (a) The Corollary shows that the evaluation mapping associated with a reflexive group is continuous if and only if the group is locally compact. This result has recently been found by E. Martín-Peinador ([7]). Her proof is based on the concept of convergence groups. Our approach is much simpler.

(b) Neither of the two properties from part (iii) of the Theorem implies the other. In order to see this let us first consider any reflexive group $G$ which is not locally compact. (For example, we could choose $G$ to be the additive group of some infinite dimensional Banach space.) Then $\alpha_{G}$ is continuous while $G^{*}$ is not locally compact.

Next we present a class of groups $G$ such that $G^{*}$ is locally compact and $\alpha_{G}$ is not continuous. Moreover, $\alpha_{G}$ is open and bijective. Let $H$ be a non-compact locally compact abelian group and let $G$ denote the group $H$ endowed with the weak topology, i.e., the weakest topology such that all characters of $H$ are continuous. We prove that $G$ is a topological group which satisfies the properties mentioned above. First let us note that the mapping $\varphi: H \rightarrow \mathbf{T}^{H^{*}}, x \mapsto(\chi(x))_{\chi \in H^{*}}$, induces a topological isomorphism of $G$ onto $\varphi(H)$. (In particular, $G$ is a topological group.) If $G$ is locally compact, then $\varphi(H)$ is a closed subgroup of the compact group $\mathbf{T}^{H^{*}}$ and hence is compact. Thus $G \neq H$ (as topological groups). From a theorem 
of Glicksberg ([4],1.2; cf. [2]) it follows that $G^{*}=H^{*}$ (as topological groups). In particular, $G^{*}$ is locally compact and $\alpha_{G}=\alpha_{H}$. Since $\alpha_{H}: H \rightarrow H^{* *}$ is a topological isomorphism, it follows that $\alpha_{G}$ is an open isomorphism and $\alpha_{G}$ is not continuous.

(c) The following examples show that the Corollary is no longer valid if one of the hypotheses on $\alpha_{G}$ is omitted. Suppose that $G^{*}$ is discrete. Then the evaluation mapping is continuous and $\alpha_{G}$ is continuous. First we consider a non-trivial group $G$ such that $G^{*}$ is trivial. Then $\alpha_{G}$ is open and surjective. Moreover, $G$ is not reflexive and hence is not locally compact. As a standard example for groups with trivial character groups, we mention $L^{p}$-spaces with $0<p<1$ ([5],23.32). For further examples see [11]. Dierolf and Warken proved that every abelian Hausdorff group is a closed subgroup of an abelian Hausdorff group without non-trivial characters $([3])$.

Now let $G$ be the torsion subgroup of $\mathbf{T}$. Note that $G$ is not locally compact. The inclusion mapping $\iota: G \rightarrow \mathbf{T}$ is an injective character of $G$. In particular, $\alpha_{G}$ is injective. It is known (cf. the last lines of [9]) that the mapping $k \mapsto \iota^{k}$ is a topological isomorphism of the discrete group of integers onto $G^{*}$.

\section{ACKNOWLEDGEMENT}

I am indebted to Lydia Außenhofer for an inspiring discussion which led to the present note.

\section{REFERENCES}

1. W. Banaszczyk, Additive Subgroups of Topological Vector Spaces, Springer Lecture Notes in Mathematics 1466, 1991. MR 93b:46005

2. W.W. Comfort, F.J. Trigos-Arrieta, Remarks on a theorem of Glicksberg, pp. 25-33 in: General topology and applications, Proceedings of a conference in Staten Island, 1989, (Eds.: S.J. Andima, R. Kopperman, P.R. Misra, J.Z. Reichman, A.R. Todd), Dekker, New York, 1991. MR 92k:54042

3. S. Dierolf, S. Warken, Some examples in connection with Pontryagin's duality theorem, Arch. Math. 30 (1978), 599-605. MR 58:16943

4. I. Glicksberg, Uniform boundedness for groups, Canad. J. Math. 14 (1962), 269-276. MR 27:5856

5. E. Hewitt, K.A. Ross, Abstract Harmonic Analysis I, 2nd ed., Springer, 1979. MR 81k:43001

6. S. Kaplan, Extensions of the Pontrjagin duality I: Infinite products, Duke Math. J. 15 (1948), 649-658. MR 10:233c

7. E. Martín-Peinador, A reflexive admissible topological group must be locally compact, Proc. Amer. Math. Soc. 123 (1995), 3563-3566. MR 96a:22002

8. S.A. Morris, Pontryagin Duality and the Structure of Locally Compact Abelian Groups, Cambridge University Press, Cambridge 1977. MR 56:529

9. N. Noble, k-groups and duality, Trans. Amer. Math. Soc. 151 (1970), 551-561. MR 42:4963

10. V. Pestov, Free abelian topological groups and the Pontryagin - van Kampen duality, Bull. Austral. Math. Soc. 52 (1995), 297-311. MR 96k:22002

11. D. Remus, Topological groups without non-trivial characters, pp. 477-484, in: General Topology and its Relations to Modern Analysis and Algebra VI, Proc. Sixth Prague Topological Symposium 1986, (Ed.: Z. Frolik), Heldermann Verlag, Berlin, 1988. MR 89f:22002

12. M.F. Smith, The Pontrjagin duality theorem in linear spaces, Ann. of Math (2) 56 (1952), 248-253. MR 14:183a

Mathematisches Institut, Universität Tübingen, Auf der Morgenstelle 10, D-72076 Tübingen, Germany

E-mail address: gerhard.turnwald@uni-tuebingen.de 